\title{
A Practical Approach to Monitor Capacity under the CDM Approach
}

\author{
Catya Zuniga ${ }^{*}+$ and Geert Boosten $^{\dagger}$ \\ Aviation Academy, Amsterdam University of Applied Sciences, 1097DZ Amsterdam, The Netherlands; \\ g.boosten@hva.nl \\ * Correspondence: c.a.zuniga@hva.nl \\ † These authors contributed equally to this work.
}

Received: 21 April 2020; Accepted: 14 July 2020; Published: 21 July 2020

\begin{abstract}
The operations of take-off and landing at hub airports are often subject to a wide variety of delays; the effects of these delays impact not only the related stakeholders, such as aircraft operators, air-traffic control unity and ground handlers but as part of the European network, delays are propagated through the network. As a result, Airport Collaborative Decision Making (A-CDM) is being employed as a methodology for increasing the efficiency of Air Traffic Management (ATM), through the involvement of partners within the airports. Under CDM, there are some strategic common objectives regardless the airport or the partner specific interest to improve operational efficiency, predictability and punctuality to the ATM network and airport stakeholders. Monitoring and controlling some strategic areas such as, Efficiency, Capacity, Safety and Environment is needed to achieve the benefits. Therefore, the present work aims to provide a framework to monitor the accuracy of capacity in the three main flight phases. It aims to provide a comprehensible and practical approach to monitoring capacity by identifying and proposing Key Performance Indicators (KPIs) based on the A-CDM Milestone Approach to optimise the use of available capacity. To illustrate our approach, Amsterdam Airport Schiphol is used as case study as a full A-CDM airport.
\end{abstract}

Keywords: A-CDM; milestone approach; demand; capacity; strategic objectives

\section{Introduction}

Different airports in Europe and around the world are working under the Airport Collaborative Decision Making (A-CDM or CDM) principles due to its benefits; this aims to improve operational efficiency, predictability and punctuality to the Air Traffic Management (ATM) network and airport stakeholders' operations by working under an ATM network [1]. A-CDM aims to improve network operations through quality and timely information exchange, procedural improvements, collaborative decision support tools, and common situational awareness. A-CDM is based on a change of mind-set and working process involving the main stakeholders of an airport. It is an approach that allows airlines, airport operators (AO), ground handlers (GH), air-traffic control (ATC) and other stakeholders to exchange operational information and work together to manage airport operations by optimizing the use of resources and improving predictability of key events of the flight [2].

A-CDM brings significant benefits to all partners establishing the mechanisms to improve the quality of information on which decisions are made which lead to enhanced operational efficiency and facilitate optimum use of available capacity [1]. It is not hard to see that different partners will have different views on what are their success factors and needs, nevertheless, there are some success factors of A-CDM, regardless the airport or the partner specific interest:

- Develop common situational awareness by sharing accurate and on-time information; 
- Develop standardized vocabulary, definitions and processes;

- Use one shared airport information platform feed by standardized data of operations;

- Develop tools and procedures for normal and non-normal operations that are acceptable to all partners.

To achieve these goals, it is necessary to define clear and achievable objectives and follow and manage the course of their results, i.e., to monitoring and manage the performance on certain key areas such as predictability. Therefore, this work aims to provide an understandable and practical approach to monitoring capacity as one of the main strategic objectives set collaborative by the airport partners. This framework is in line with the A-CDM principles and Total Airport Management (TAM) principles ones. CDM provides the foundation to build an airport's operational communication protocol to show the performance of operations to the partners; it enables the use of data across the entire airspace, airport's airside and landside operations, both in real-time operations but also as post analysis tool for reviewing performance of operations and a tool for proposing or follow controlling actions. The possibilities for gains on performance knowledge by analyzing these data information, not just at the airport but across aviation partners is crucial.

In addition, this work presents a brief review on performance metrics and indicators for capacity which are the baseline for the selection of the key performance indicators (KPIs) to monitor capacity on pre-tactical phase both at macro and micro level using six main reference points on the landing and take-off cycle (LTOC). The six reference points use the milestone approach and its associated data sets as input parameters for traffic movements, both the actual and the forecasted or estimated demand.

To illustrate our approach, Amsterdam Airport Schiphol (ICAO code EHAM) is used as case study. It is the main airport of the Netherlands and the 3rd busiest airport in Europe moving on 2017 about 68.5 million passengers. The CDM program at Schiphol (CDM@SPL) has been an initiative between the airport, the Dutch Air Navigation Service Provider (LVNL), their operating airlines and ground handlers, see [3]. CDM@SPL has been implemented in two phases. In the first phase, Schiphol started local operations according to the CDM method, basing its operational decisions on shared information concerning the flight and aircraft handling process that it receives from the airport, air traffic control, airlines and ground handling. In the second phase, Schiphol started to share its operational information with Eurocontrol in real time, being the 28th full A-CDM airport connected to the network manger's system aiming to ensure more efficiently scheduled time slots for flights to and from Schiphol, which will in turn promote more efficient management of the Dutch and European airspace.

The organization of this work is as follows, Section 2 introduces background information about capacity and the performance indicators to address it. Section 3 address briefly the CDM concept elements as part of the background of the problem, together with the milestone approach address in Section 3. Section 4 presents describes the indicator about accuracy of declared capacity, providing deeper information about the case study of CDM program at Schiphol, explaining the generalities of the operations on the different phases of the flights; meanwhile, Section 5 describes the proposed approach for analyzing with more detail the accuracy of operations by analyzing diverse time reference and events on the Milestone approach. Finally, conclusions and further research is presented.

\section{The Basics about Capacity and Its Performance}

Performance measurement and management helps organizations all around the world, whether they are multinationals, government departments, or small local charities to recognize the organization's performance by measuring the key success factors according to the strategic objectives. In the last two decades, performance has been one of the major keywords for aviation as well as for many organizations. Performance-based management (PBM) places attention on results, responsibilities, and targets; performance indicators (PI)s are used by managers to allocate assets or to establish which strategy to implement to achieve the desired goals, these indicators can also be used as communication protocol to show the performance of operations to the outside world. PBM integrates existing financial, operations and other data into eye-opening and actionable facts for enlightened decisions. It is able to consistently track cost and performance over time and improve predictability. In [4], PBM is defined as a systematic 
approach to performance improvement through an ongoing process of establishing strategic performance objectives; measuring performance; collecting, analyzing, reviewing, and reporting performance data; and using that data to drive improvement.

Similar to PBM, other proposals can be found in the performance management area, such as the performance measurement systems which are responsible for coordinating indicators across the various functions, and for aligning the indicators from the strategic (top management) to the operational levels. For every activity/product/function, multiple indicators can be developed and implemented. The challenge is to design a structure for the indicators (i.e., grouping them together) and extracting an overall sense of performance from them [5]. A helpful review of performance measurement systems can be found in [6] where it is presented different PMSs which have been developed to be used in companies.

Regarding the ICAO, the concept of performance management system $[7,8]$ is to provide managers with real-time information on the degree of fulfilment of the set objectives, and hence with reliable support in decision-making process; this system includes three basic functions: performance measurement, performance monitoring and performance review. This applies to the ATM system which has to meet strict performance criteria, particularly in the area of safety. The main advantages of PBA are the orientation to results and meeting customer expectations; promotion of greater accountability among managers; the adoption of business strategies becomes much more transparent, because the goals to be achieved are clearly defined and quantified. Other benefits which are worthy to mention are the greater freedom and flexibility in the choice of solutions, leading to greater cost-efficiency; better allocation of resources; and better predictability.

Although there are some variations of this process, they are all based on a similar philosophy and principles $[7,8]$. Step 1 -Define/review scope, context and general ambitions and expectations; Step 2-Identify opportunities, issues and set (new) objectives to decide which specific performance aspects are essential for meeting the general expectations; Step 3-Quantify objectives to ensure that the set objectives are specific, measurable, achievable, relevant and time-bound (SMART); Step 4-Select solutions to exploit opportunities and resolve issues, this step can be divided into three sub-steps: selection of the decisive factors to reach the target performance, identification of solutions to exploit opportunities and mitigate the effects of the selected drivers and blocking factors and selection of sufficient set of solutions; Step 5-Implement solutions and Step 6-Assess achievement of objectives.

The total airport management (TAM) principles enabling airport partners to maintain a joint plan-the airport operations plan-thus working towards dynamically agreed goals in order to get full CDM benefits. This implies the extension of the tactical time horizon to pre-tactical and strategic phases. The scope of TAM is the entire airport, monitoring and guiding airside and landside operations while taking into account additional information available $[9,10]$. TAM is a performance-based airport operations approach enabling performance-based air traffic management; it is aligned with existing concepts and systems but integrates them into one holistic architecture, and it provides a platform whereby operational decisions taken by partners may be made in the full knowledge of the other partners in key moments.

Along these lines, the work presents by [11,12], introduces an advanced approach of performance-based airport management (PBAM), developed by combining the existing concepts of TAM and performance-based management. The Performance-Based Airport Management is introduced to close the gap between performance target and day-to-day operations. The general idea behind is a collaborative coordination and control of airport operations based on defined performance targets. In this work, CDM and Total Airport Management principles are included. PBAM is achieved by five steps: (1) identifying long-term Targets, per partner and on system bases on a collaborative manner, (2) identification of the key performance indicators (KPIs) and metrics, (3) continuous measurement monitoring and prediction of operations, (4) Aggregated KPI results from the lower levels provide the basis for the KPI value determination of the higher level. (5) Actions needed after evaluating the performance 
from project team, senior management, external project facilitators, and team coordinators whose role it is to help the strategy to success

Therefore, in this work it is proposed to use the PBM incorporating PBAM and TAM principles to define a systematic approach to monitor the performance through the following steps:

- Identifying strategic performance objectives and long-termtargets in line with the European, regional and local principles;

- Measuring performance of operations, by the identification of the proper roadmap and goals to achieve strategic objectives and targets;

- Identifying Timeframe, KPIs and Metrics suitable to represent and regulate a specific process function and goal;

- Define set of actions needed after evaluating the performance as a management tool to encourage timely action in the right direction;

- Continuously monitoring and managing of performance using that data to drive improvement.

Performance indicators (PIs) provide a means of "distilling" the larger volume of data collected and can be used both to judge outcome performance and to predict future performance, KPIs are current- or future-oriented measures; they should be monitored 24/7, daily, or perhaps weekly for some, mainly because they are key to the business. The terminology used in this context is not completely and univocally defined, hence, special attention is placed on the terminology proposed by the TAM and CDM principles. Often, similar concepts are classified using different terms, depending on the technical area of interest, hence, in this work, some definitions are stated.

It is possible that one of the most difficult activities in PBM is making systems "tangible" by means of their performances indicator. Process managers try to do this, translating organization goals into different metrics (indicators), which are also visible from the outside. Hence, a specific literature review was conducted to identify the proposed KPIs and metrics regarding Capacity.

According to ICAO [7,8,13], Capacity can be measured either system-wide, airspace and/or airport.

On system-wide or network bases, measuring capacity can be performed by monitoring diverse KPIs such as the number of flights, flight hours and flight distance that can be accommodated on certain period of time; or by the number of flights, available plane miles, etc. For the airspace, the identified capacity KPIs are the number of IFR flights able to enter an airspace volume, or by the number of IFR flights able to be present in sectors at any one time (airspace capacity rates). It has been highlighted that it is key to monitor the ability to objectively determine the number of flights able to enter an airspace volume. Finally, the airport KPIs mentioned are by ICAO are the hourly number of IFR movements (departures plus arrivals) as possible during low visibility conditions, daily number of IFR movements (departures plus arrivals) as possible during a 15-h day between 7:00 and 22:00 local time during low visibility conditions $[13,14]$.

The KPIs defined by the Single European Sky ATM Research (SESAR) 2020 Performance Framework, regarding capacity are divided into three areas of performance: Airspace Capacity, Airport Capacity and Capacity Resilience [14]. Airspace Capacity's KPIs proposed are two: Terminal Maneuvering Area (TMA) throughput in challenging airspace, per unit time and En-route throughput in challenging airspace, per unit time. Airport Capacity is mainly measure by the Peak Runway Throughput (Mixed mode). The Capacity Resilience has two main KPIs, loss of airport capacity avoided, and loss of airspace capacity avoided (represented on percentage). In [12], it can be found a very interesting review of the current KPIs and PIs used by different partners and agencies; several Key Performance Areas are covered and their specific KPIs and PIs used in Europe are described.

The Civil Air Navigation Services Organization published 21 operational KPIs that allow Air Navigation Service Providers (ANSPs) to track targeted areas of their systems, regarding ATM Capacity, three main KPIs are suggested: the Declared Capacity defined as the target acceptance rate for a facility or sector; the Capacity Efficiency represented by the percentage of demand accommodated by facility's 
capacity and actual demand; and the Delay Attributed to Capacity Limits or Total or Average Delay by Airport [14,15].

To successfully capture a trade-off between partners objectives the identification of airport performance objectives and stakeholder drivers or incentives over the course of the flights' LTOC is required. Therefore, and due to its nature, CDM proposes both system/network and partners' common objectives and performance metrics between all A-CDM stakeholders, based on mutually agreed targets. Under the CDM principles the four main objectives are Efficiency, Capacity, Safety and, Environment [1]. A wide variety of information about the CDM concept, benefits, implementation, management and more can be found on the CDM at Eurocontrol, the Civil Air Navigation Services Organisation (CANSO) [16] and the ICAO website [9].

The Airport Capacity Assessment Methodology guidance material, [17] proposes metrics, its main pros and cons, and measures regarding Airside Capacity, together with the interfaces between the runway and TMA where the approach flows of aircraft elapses with other activities on the ground such as those in the gates and stands. In this manual, some interdependencies between the Airspace Capacity and Landside Capacity are mentioned particularly for those areas that overlap between the domains. Three main types of capacity are identified: the Structural Capacity which provides input to early Air Traffic Flow and Capacity Management (ATFCM) processes and its time frame starts years before day of operations and until some months; depending on the business model and size of an airport this can range every 2 to 5 years to an annual basis. This capacity provides the airport management with its first input for both business case decision making and to identify the requirements for further detailed planned capacity analysis. The Planned Capacity is used by all airports for coordination on airline's schedule (with or without the worldwide slot guidelines process) and also as input to the strategic flow management processes with the Network Manager. It is calculated at least 18 months prior to the season (summer and winter) for coordinated airports and closer to the season as identified by the local stakeholders for the other group of airports. And the Operational Capacity which is used as part of the Network Strategic, Pre-Tactical and Tactical processes up to the day of operations. Furthermore, for addressing the influencing factors and assumptions, more detail types of capacity are mentioned: the Runway Operations Capacity, Taxi Operations Capacity, and the Apron/Gate Operations Capacity. Hence, the Structural Capacity, Planned Capacity and Operational Capacity mainly are mainly used to indicate that each airport element (i.e., runway, taxi system, aprons and gates) has different input parameters depending on the timeframe, the closes to the day of operations, the more detail input on different parameter is required for planning purposes.

It can be concluded that numerous approaches in the literature frequently estimate capacity as the maximum number of aircraft an Air Traffic Control (ATC) unit can handle at a given time, referring to airspace capacity. This work does not aim to provide an extensive overview of those definitions but rather point out the complex nature of capacity, its drivers and characteristics. Capacity is dynamic and interactively influenced by many factors or drivers. In addition, the selection of KPIs should consider not only technical drivers, but other important ones, such as the social factors and the business model of airlines and airports among other stakeholders.

\section{The Airport Collaborative Decision Making (A-CDM) Principles}

The CDM principles are performing successfully collaborative processes including standardized communication protocols, procedures, tools, and information sharing to move operations into a collaborative management process that improves overall system performance and benefits the individual stakeholders. To accomplish the above-mentioned goals, a solid base on transparency and information sharing is needed to the establishment of collaborative working methods and practices which will be used for monitoring purposes. The A-CDM proposes five basic elements, specially the Information Sharing and the Milestone Approach, as a key to improve efficiency and predictability. Information Sharing is implemented using a shared airport information platform among partners. Once in place the information sharing mechanism, the Milestone Approach can take place helping 
an early and accurate prediction for $\mathrm{ATC}, \mathrm{AO}$, and $\mathrm{GH}$ operations and hence, to anticipate resources needed to cope with the situation. For this, specific milestone events are monitored, and trigger alerts to prompt awareness if a problem occurs, helping partners on the decision-making process for downstream events which influence the further progress of the flight $[1,18]$.

The milestones represent significant events that occur during the LTOC that occur during the planning or operation of a flight. The milestone approach is based on 16 general processes that describe the progress of a flight on three main phases called, inbound, turn round normally called (turnaround) and outbound. Monitoring these milestones both in a strategic, pre-tactical and tactical level, allows partners to anticipate problems quickly when there is any deviation from the plan. In pre-tactical level, monitoring the performance of certain indicators help to develop tools and procedures that are acceptable to all partners on how to act on normal and disruptive situations to achieve strategic objectives. The following information is mainly taken from $[1,2,16,18]$.

\subsection{CDM Concept Elements}

To begin with, the element that triggers the cycle of the Airport CDM concept elements is Information Sharing. CDM is successful when all airport partners (airport operators, aircraft operators, ground handlers, Air Traffic Control and Network Manager Operations Center (NMOC)) are transparent and share their available information accurately and on time. Information sharing is essential for the other elements and must be implemented first; it is the key to creating a common awareness of each individual flight and the overall situation at the airport. Once the information is available for all stakeholders, the cycle of the CDM concept elements can be implemented, as it can be seen in Figure 1: the milestone approach, variable taxi time, (collaborative) pre-departure sequence, CDM in adverse conditions and collaborative management of flight updates.

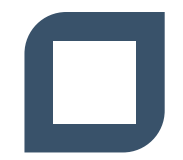

THE MILESTONE APPROACH

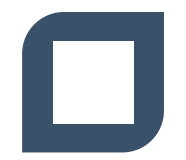

VARIABLE TAXI TIME

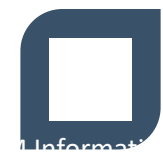

(COLLABORATIVE) PREDEPARTURE SEQUENCE

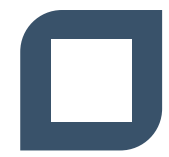

CDM IN ADVERSE CONDITIONS AND

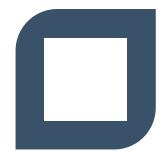

COLLABORATIVE MANAGEMENT OF FLIGHT UPDATES

Figure 1. The airport collaborative decision making (CDM) concept elements own elaboration, Source: [1].

The milestone approach is recommended to be implemented as a baseline on new processes and procedures where collaboration between people of all partners is the central concept. Due to its importance, further description will be provided in this work. The second element of the cycle is about the variable taxi time (VTT) which helps to gain more accurate estimates of in/off-blocks and take-off operations. With VTT in place, the link between off-block time and take off time becomes transparent to all partiers and network operations, and a proper prediction of the take-off time can be communicated towards the network function represented by network operations.

The third element is about building a (collaborative) pre-departure sequence; its aim is to regulate the order for departing aircraft based on the off-block time and considers operational constraints and preferences of the operators. With the Pre-Departure Sequence Planning, the order in which a flight may push-back or start the engines is regulated with the expected start-up approval time issue by ATC. The given start-up approval time is based on the CDM principle of "best planned, best served" which overrules the "first come, first served" principle which was more common before CDM was implemented. The benefits of Pre-Departure Sequence Planning are to increase the punctuality and improves the slot-adherence while considering the preferences of the airport partners.

The fourth element is about CDM in adverse conditions which are defined as "Adverse conditions consist of collaborative management of the capacity of an airport during periods of predicted or 
unpredicted reduction of capacity". To make collaborative decisions during adverse conditions, an $\mathrm{CDM}$ cell is introduced. In adverse conditions, the goal is to create a common situational awareness for each of the airport partners, better information could be shared as all communication lines are short. With better information, passengers could be informed correctly, and the disruption could be better anticipated and, therefore, better planning of resources.

The fifth element is about the collaborative management of flight updates which helps to have better quality of information regarding arrival and departure information. The information is exchanged by the network operations, with all CDM airports to have a better predictability for airspace capacity, and hence, to plan required resources in an efficient way. One feature element is the execution of the ground delay program, this is performed by the network manager and issue through a departure time stamp calculated by the appropriate central management unit, as a result of tactical slot allocation, at which a flight is expected to become airborne.

\subsection{The Milestone Approach}

The Milestone Approach can be divided into three main phases (see Figure 2) which are:

- "Inbound or arrival", including the milestones: M1 until M6;

- $\quad$ the second phase is called "Turn round" and it includes M7 until M14; and

- the "Outbound" phase, including M15 and M16.
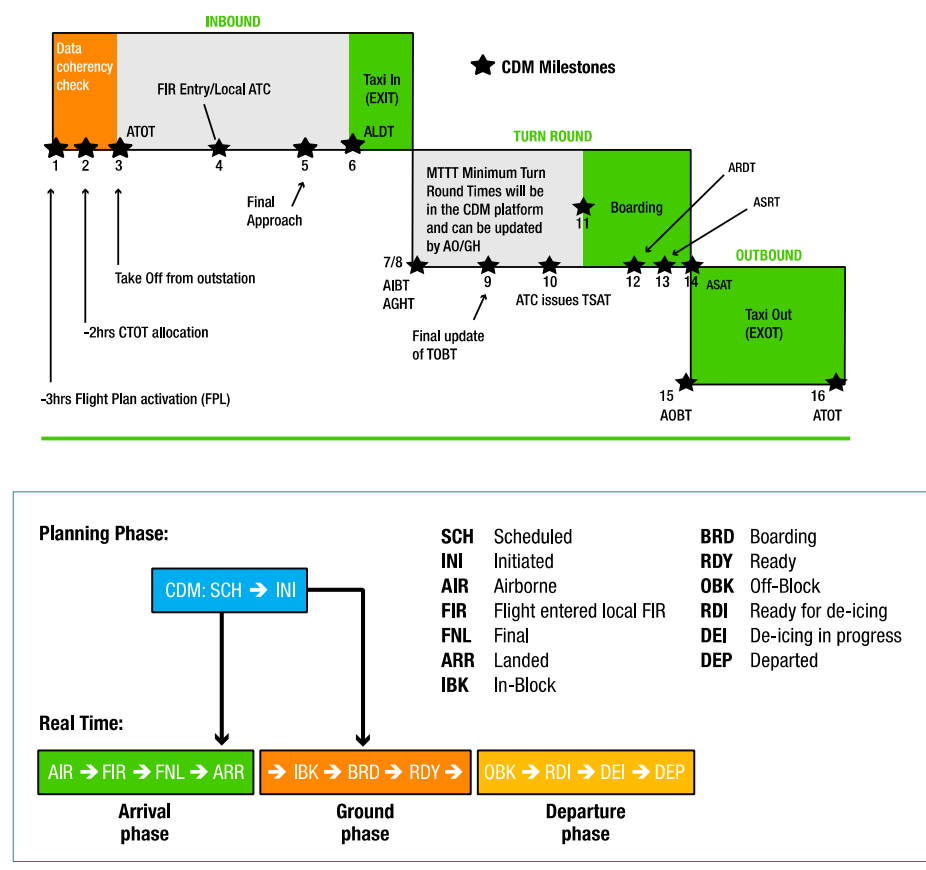

Aircraft flight status: in addition the ground radar picture used by ATC (e.g. A-SMGCS) can be shared by CDM partners so that the location of aircraft and vehicles can be monitored on the aerodrome surface

Figure 2. The milestone approach and its nomenclature. Source [1].

The inbound phase covers all the activities from the moment the flight plan is activated until the aircraft passes the red line at the destination airport's apron, which is equal to the operational status 'On-Blocks', see Figure 3. The first two milestones (Milestone 1 and 2) are mainly aiming to initiate CDM platform for each flight and check consistency on flight data for planning purposes. Since this moment different time reference estimations are performed related to landing, in/off-block and take-off time, in addition to issue some regulations through the Calculated Take-Off Time. The milestone 3 is called "Take-Off from Outstation" and it is achieved at a time reference called Actual Take Off Time 
(ATOT). From the moment the flight is within on average $40 \mathrm{~min}$ and even $3 \mathrm{~h}$ flying time of the destination airport the Network Operations monitors progress of the flight sending a Flight Update Message (FUM) that provides updates of the flight's progress until the flight enters the FIR (Flight Information Region) or the local airspace of the destination airport, mark with the Milestone 4.

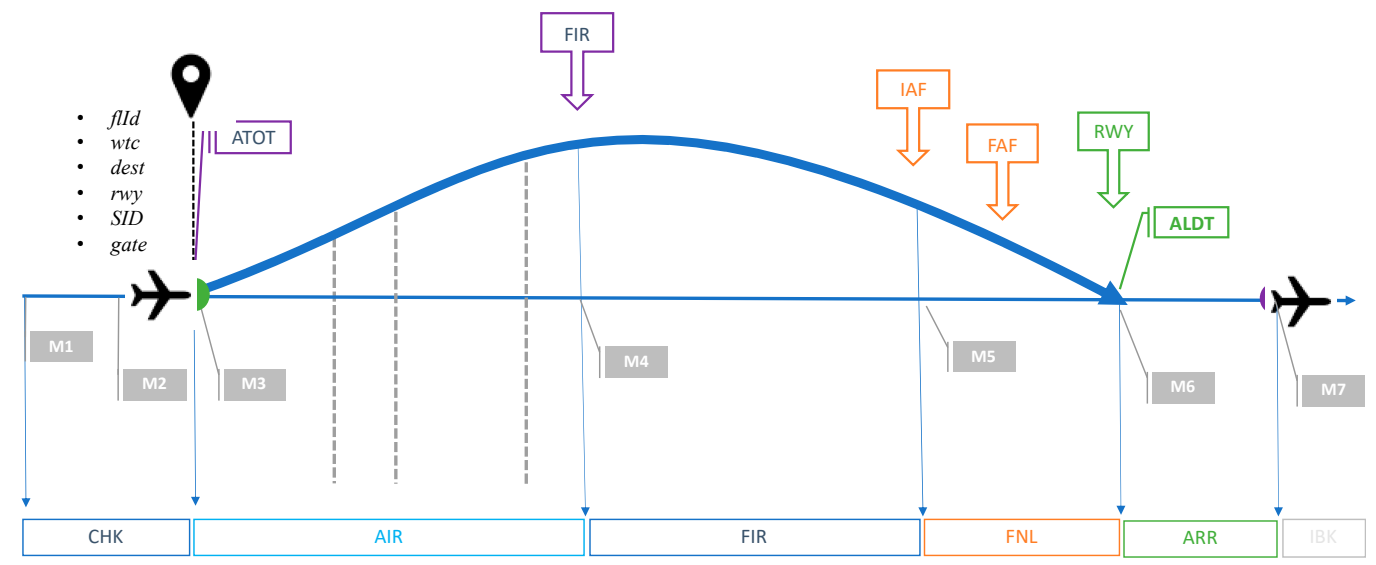

Figure 3. The inbound phase of the flight on the milestone approach and its nomenclature.

In Milestone 4 and 5, important updates on the Estimated Landing Time (ELDT) and Target Off-Block Time (TOBT) are performed; hence, different updates on information of the progress of the flight are needed to update the estimated landing and off-block moments. The accuracy of ELDT is quite important at this stage since downstream decisions are taken based on this two key moments; these decisions concern all partners, such as stand or gate utilization, aircraft changes, preparation of arrival sequence, preparation of ground-handling operations, or decisions for connecting passengers, to mention some.

The TOBT is defined as the time that an AO or GH estimates that an aircraft will be ready, all doors closed, boarding bridge removed, push back vehicle available and ready to start up and push back immediately upon reception of clearance from the TWR for next flight. A key requirement for TOBT assignment is accuracy because a reliable TOBT allows us to calculate air-traffic flow management (ATFM) slot delay and issues a Target Start-up Approval Time (TSAT) to inform the flight crew and all partners about the time when the aircraft can expect start up and/or pushback approval. Finally, as part of the arrival phase Milestone 6, where an aircraft touches down on a runway at the so called Actual Off-block time (AOBT); equivalent to ATC Actual Time of Arrival (ATA). The actual landing of the aircraft also triggers several updates, but it can be highlighted, the moment of off-blocks and take-off, represented by TOBT and Target Take Off Time (TTOT) which are updated by the Aircraft Operator/Ground Handler, calculated on the basis of the defined turn-round period and estimated taxi-out for the departing flight.

As shown in Figure 4, the Turn-Round phase is determined when the aircraft is in-block until it is off-block, represented by the Actual In-Block Time (AIBT) and the AOBT, respectively. Once the aircraft is in-block (Milestone 7), the ground-handling operations start (Milestone 8). The process that takes place during this turnaround time comprises of the planning and handling of tasks to ensure the cleanliness, safety and efficiency of the next flight. Therefore, the turnaround time is one of the most important milestones for airport operators, directly affecting whether or not passengers will enjoy a timely optimal experience.

To achieve these different reference times the TOBT and the TSAT are updated, on Milestone 9. As previously mentioned, the TOBT represents the time that an airline or handling agent estimates that an aircraft will be ready, all doors closed, boarding bridge removed, push back vehicle available, ready to start up/push back immediately upon reception of clearance from the tower; TSAT is the time ATC issues the TSAT taking into account TOBT, CTOT and/or the traffic situation that an aircraft can expect start-up/push back approval. 


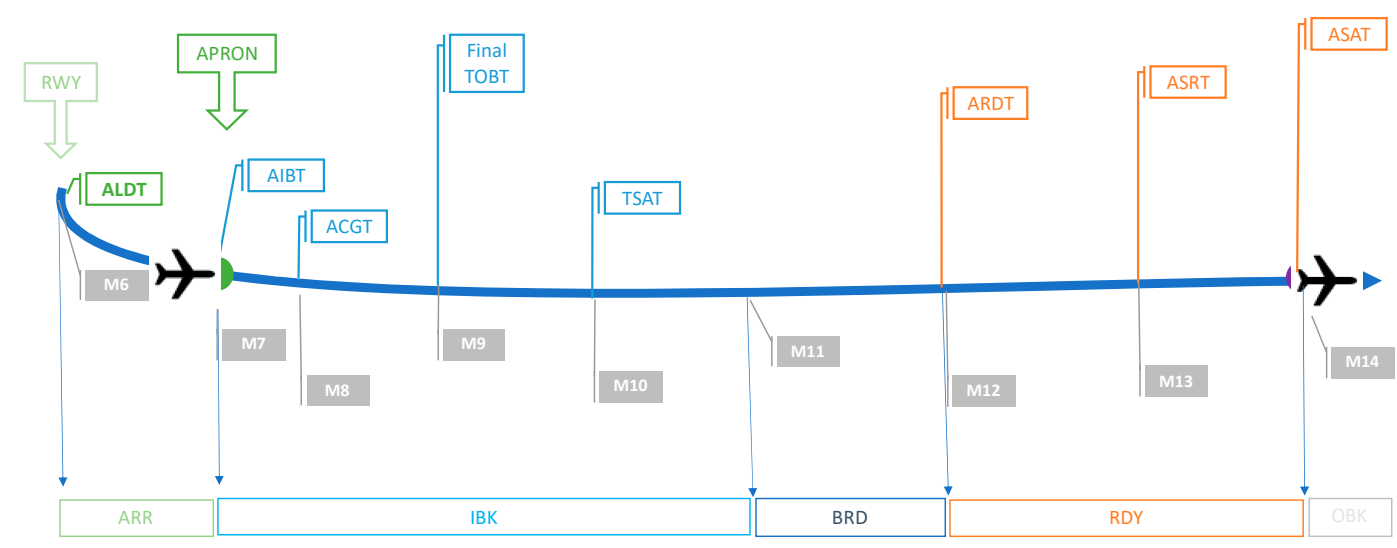

Figure 4. The turn-round phase of the flight on the milestone approach and its nomenclature.

Once the boarding process is completed at the so-called time reference Actual Ready Time (ARDT) defined as the moment when the aircraft is ready for start-up/push back or taxi immediately after clearance delivery, meeting the requirements set by the TOBT definition; the pilot can request for clearance to ATC. Therefore, it is the responsibility of ATC to inform the flight crew and other partners when an aircraft can expect to receive start-up and/or pushback approval which takes into account the TOBT as well as the allocated ATFM slot and the traffic situation, this will happen at the Actual Start-Up Approval Time (ASAT).

The outbound phase starts when the aircraft is pushed back from the apron or vacates its parking position at the so called Actual Off-Block Time (time reference at AOBT), see Figure 5. The aircraft starts rolling from the standpoint (or detachment point) using certain taxiways that Ground ATC instructs the aircraft to go through until its assigned runway. This phase will be call taxi-out phase and it involves all activities from the moment the aircraft is actually off-block such as de-icing, waiting on crossing hot spots or taxi-routes, but also the aircraft holding at runways or lining up. The aircraft rolls until the "holding point" entering the runway. Normally, ground controls change here to tower control. Depending on the traffic conditions both on the ground and on the air, the aircraft can continue to line-up or wait at holding point until getting clearance from controllers. This process called "multiple line-up", can be done in parallel with other aircraft if the local procedures allows. Once the aircraft enters the runway to line-up, it is ready for rolling to departure. This subprocess finishes at Actual Take-Off Time (marked by the time reference ATOT); which is the time that an aircraft takes off from the runway time, equivalent to airline/handlers' Actual Time of Departure (ATD). After that, the aircraft departs climbing until certain point (typically $30,000 \mathrm{ft}$ or $10 \mathrm{~km}$ ) before it can cruise at this altitude in a safe and economic way to continue its en-route phase.

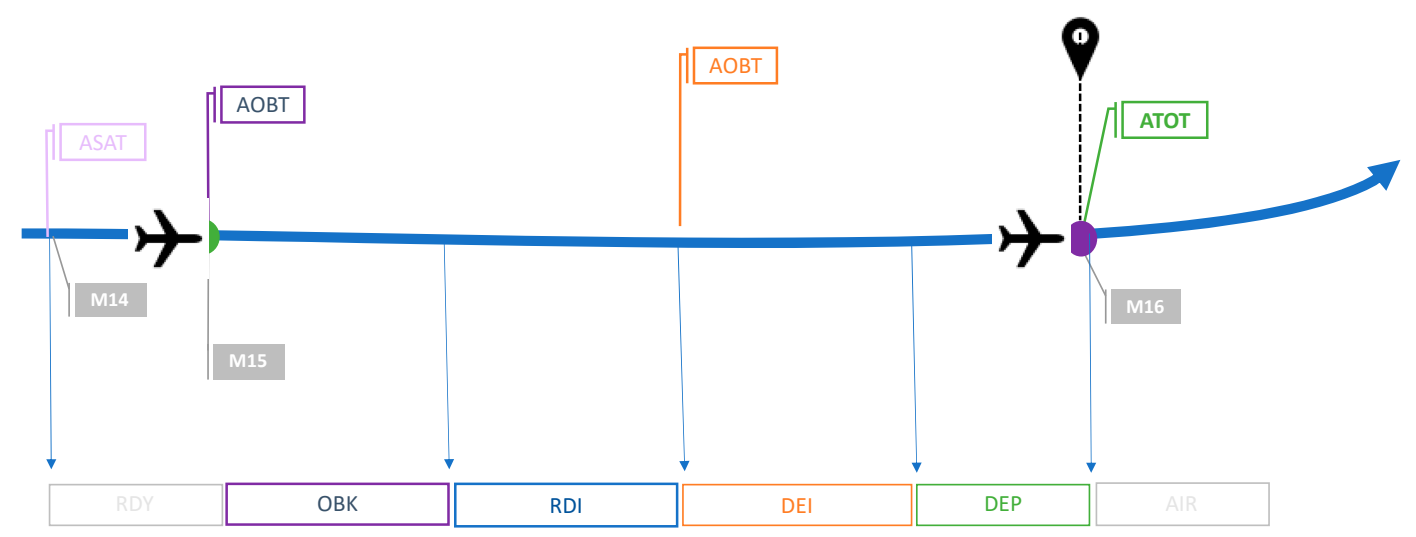

Figure 5. The outbound phase of the flight on the milestone approach and its nomenclature. 


\section{Measuring the Capacity of Operations}

This work aims to propose the use of the KPI called Accuracy of declared capacity found in [1] as a baseline to generalize the KPI for pre-tactical purposes and referring to a different simple and standard manner to address capacity in an organize and efficient manner. Monitoring the LTOC by each phase of flight, allow us to gain a deeper understanding of key success factors affecting capacity, not only at the airport or airspace level, but at network level. The propose framework aims to monitor capacity in such a way that the success factor, drivers and business models per each of the flight are identified.

The definition of accuracy is essentially having results (data) that is centered on a target value. Statistically speaking, it is how correct the mean value is of your estimations with respect to the actual operations. Therefore, the performance indicator known as Accuracy of declared capacity should be measure by comparing the actual number of movements (realization) to the declared capacity, see (Equation (1)):

$$
A c c_{d}=d_{a}-d_{e}
$$

where,

$A c c_{d}$ is the accuracy of declared capacity;

$d_{a}$ is the actual traffic movements (realization) at certain time interval; and,

$d_{e}$ is the declared capacity.

The declared capacity at Schiphol Airport is determined in a cooperation [19]; they specify the declared capacity as "the highest substantiated capacity per hour during a "mode" of the ATM system which can be achieved depending on the runway combination" (see Table 1). The declared Capacity is defined as the "capacity number independent of the runway combination that can be delivered with a certain reliability under normal circumstances. This number is determined by the sector and is used for slot allocation".

Table 1. Declared capacities at Schiphol Airport. Source: [19].

\begin{tabular}{cccccc}
\hline Operational Mode & Local Time & \multicolumn{2}{c}{ Nominal Hourly Capacity } & \multicolumn{2}{c}{ Nominal Capacity per 20 min } \\
\hline & & Inbound & Outbound & Inbound & Outbound \\
\hline Outbound peak & $07: 00-22: 40$ & 36 & 74 & 12 & 25 \\
Inbound peak & $07: 00-22: 40$ & 68 & 38 & 23 & 13 \\
Off peak mode & $06: 00-22: 40$ & 24 & 30 & 8 & 10 \\
& $06: 40-07: 00$ & 24 & 40 & 8 & 14 \\
& $07: 00-22: 40$ & 36 & 40 & 12 & 14 \\
Night mode & $22: 40-23: 00$ & 36 & 25 & 12 & 9 \\
\hline
\end{tabular}

The capacity declaration describes the coordination parameters per season and per airport with a maximum of 500,000 aircraft movements in commercial aviation during an operational year, of which no more than 32,000 are night movements (movements with a runway time in the period 23:00-6:59 LT)". In addition, the environmental and operational capacity restricts the number of operations using other parameters such as the nominal operational runway capacity for both, general and commercial aviation. Schiphol airport functions as a hub meaning that at certain times of the day there are peaks of a large amount of incoming traffic and somewhat less outbound traffic and at other times, there is more outbound than incoming traffic. It can be noted that the inbound and outbound declared capacity at Schiphol Airport presents five inbound peaks and five outbound peaks.

When Equation (1) is used for strategic purposes, many factors that affect capacity are not taken into account due to the nature of the declare capacity definition. Hence, in line with partners objective it is proposed to use Equation (1) to monitor accuracy on other expected moments on time or event, lead us to Equation (2):

$$
A c c_{t}=d_{a}-d_{t, s}
$$


where,

$A c c_{t}$ is the accuracy on an expected moment on time or event;

$d_{a}$ is the actual traffic movements in certain time interval; and,

$d_{t, s}$ is the forecasted demand in a specific moment on time $(t)$, such as entering to local airspace, flying by/through a waypoint, and produced or calculated by a given IT system (s).

It should be pointed out that, the time reference proposed by the milestone approach, both for the actual or expected demand are normally information share by all partners; depending on the implementation characteristics, big data sets can be obtained for post-operational analysis, as the one suggested on this work.

It is not hard to see that accuracy is a metric which is dependent on two terms, the actual traffic movements and the forecasted demand, being both dependent on several drivers of factors such as time, concept of operations and decision support tool or IT system used, among many. Hence, our approach defines a set of performance indicators to address the accuracy of operations as indicators at entering Dutch airspace, approaching, landing, in-block, off-block and take-off events.

For airports which have implemented CDM, especially the milestone approach, different data sets can be identify and used as historical data set of performance; furthermore, these data sets can be used by different decision support tools or IT systems used to trigger those time references.

\section{Accuracy of Operations}

\subsection{The Airspace Operations Accuracy}

The proposed KPIs in [15] regarding Airspace Capacity can be very suitable for strategic monitoring purposes; those are KPIs the Terminal Maneuvering Area (TMA) and En-route throughput. In all cases of regarding airspace capacity, it is proposed to use as estimation parameters the time references stamps produced on the CDM mechanism. Hence, the Airspace operations accuracy could be monitored through Equations (3) and (4), respectively:

$$
A C_{E R}=t h_{S E R}-t h_{E R}
$$

where,

$A C_{E R}$ is the accuracy of the En-route throughput;

$t h_{S E R}$ is the actual En-route throughput;

theR is the estimated En-route throughput at Milestone 3.

$$
A c_{L D}=t h_{S T M A}-t h_{T M A}
$$

where,

$A c_{T M A}$ is the accuracy of the TMA throughput;

$t h_{S T M A}$ is the actual TMA throughput;

$t h_{T M A}$ is the estimated TMA throughput at Milestone 4.

\subsection{The Landing and Take-Off Operations Accuracy}

The landing and take-off operations accuracy could monitor through Equations (5) and (6), respectively:

$$
A c_{L D}=t h_{A L D T}-t h_{E L D T}
$$

where,

$A c_{L D}$ is the accuracy of the landing time;

th ${ }_{A L D T}$ is the actual landing throughput (runway Throughput in mix mode);

$t_{E L D T}$ is the estimated landing throughput at Milestone 5.

$$
A c_{d e p}=t h_{A T O T}-t h_{T T O T}
$$


where,

$A c c_{\text {dep }}$ is the accuracy of the departure time;

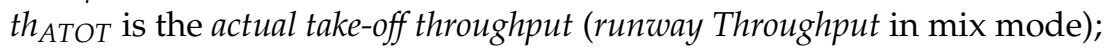

th

TTOT is the Target Take-off time, the target take-off time taking into account the TOBT/TSAT plus EXOT. This target includes remote de-icing prior to take off. The ETOT takes into account the EOBT plus Estimated taxi-out time (EXOT), i.e., the estimated taxi time between off-block and take off. This estimate includes any delay buffer time at the holding point or remote de-icing prior to take off. It should be pointed out that both EIBT and EOBT are strongly influenced by the gate-runway combination assigned. As previously mentioned, one of the A-CDM elements is devoted to analyze the taxi in/out processes, the variable taxi time, aiming to optimize the Estimated taxi-in time (EXIT) and EXOT, taking into account the taxiways layout and common procedures applied by ATC, weather changes, etc. The EXIT includes runway occupancy and ground movement time, whereas EXOT includes push back and start up time, ground movement, remote or apron de-icing, and runway holding times. These taxi times are parameters used for calculation of TTOT or TSAT and therefore, impacting on the departure sequence and take-off time.

\subsection{The In-Block Operations Accuracy}

The In-Block operations accuracy could monitor through Equation (7).

$$
A C_{I B}=t h_{A I B T}-t h_{E I B T}
$$

where,

$A C_{I B}$ is the accuracy of the in-block time;

$t h_{A I B T}$ is the actual in-block throughput;

th ${ }_{E I B T}$ is the Estimated in-block throughput at Milestone 6.

\subsection{The Off-Block Operations Accuracy}

The Off-Block operations accuracy could be monitored through Equation (8).

$$
A C_{O B}=t h_{A O B T}-t h_{T O B T}
$$

where,

$A c_{O B}$ is the accuracy of the off-block time; and,

$t h_{A O B T}$ is the Actual off-block throughput.

$t h_{T O B T}$ is the Target off-block throughput at Milestone 7.

\section{Schiphol Efforts to Monitor Operations}

As previously introduced, at Schiphol the Milestone 1,2, and 3 aim to check consistency on flight data for planning purposes related to landing, in/on-block and take-off time; and some regulations can be issued due to network restrictions/requirements (CTOTs). In this regard, research has been carried out in $[20,21]$ to address the operational benefits related to the connection of Schiphol with the network manager and the local A-CDM implementation. In [20] an analysis on procedures changes, human factors issues and operational performance. CDM effectiveness is one of the key indicator's analysis in this work; it considers take-off time predictability and non-compliance of Schiphol to take-off slots imposed by the network manager as the two primary KPIs. The research stresses that CDM has been more effective in relation to local CDM. Meanwhile in [21], local CDM analysis to improve the stakeholders' operational efficiency was performed by improving the predictability and decision making. The aim of this study is researching how local CDM has exactly improved the operation's efficiency for each involved CDM stakeholder at Mainport Schiphol, and what the exact benefits are. 
According to [22], it is worth looking at some key factors influencing the performance of the flight: aircraft entering the Flight Information Region (FIR) boundary earlier than expected, resulting in changes in the inbound, turn round and outbound expected times; an average taxi-in time is used for planning purposes with a low accuracy resulting in inaccurate estimations of in/off-block and take-off moments, in this regard, it is worth mentioning that the variable taxi time element is not yet in place at Schiphol; a significant amount on key events, such as in-block and off-block moments, updating the TOBT and turning it into a highly dynamic parameter; there is no common understanding on some terminology, definitions and meaning of vocabulary, such as the term On-time performance (OTP) is used in different ways, confusing partners' objectives.

Focusing on other aspects of the accuracy of the inbound phase, the work found in [23], based on the development a capacity management project called D-1 launched in 2018, presents an analysis of operational capacity planned before the day of operations was performed. The work aims to monitor the accuracy of the predicted traffic demand (for the departures and arrivals at Schiphol), by a comparative analysis on the expected demand forecasted a day in advance vs. the actual demand on the day of operations using data from January 2019 to March 2019. The demand is compared to different moments in time-space; when entering to the Amsterdam Flight Information Region by a specific sector; when passing by/through to its correspondent Initial Approach Fix (IAF); and, at the expected landing time calculated by Advanced Schiphol Arrival Planner (ASAP).

As can be seen on Figure 6, at approaching to EHFIRAM, the inbound flight will use five sectors to approach depending on its origin merging into three points aiming to land at a specific runway, A flight approaching the terminal maneuvering area (TMA) typically follows standard arrival routes (STARs) providing the transition from the en-route structure to terminal airspace; analogous, a flight departing will use standard instrument departure routes (SID). Depending on the airspace topology, the arrival routes merge at the initial approach fix (IAF) where the initial approach segment begins; in Schiphol, the IAFs are called SUGOL, RIVER and ARTIP, see Figure 6. To avoid potential conflicts at airspace, ATCs issues a set of vectoring instructions to pilot to overcome merging and sequencing of arrival flights. Delay in the landing times is induced if an aircraft is required to either slow down, hold, or change route to avoid potential conflicts. These manual interventions are out of the scope of [23].

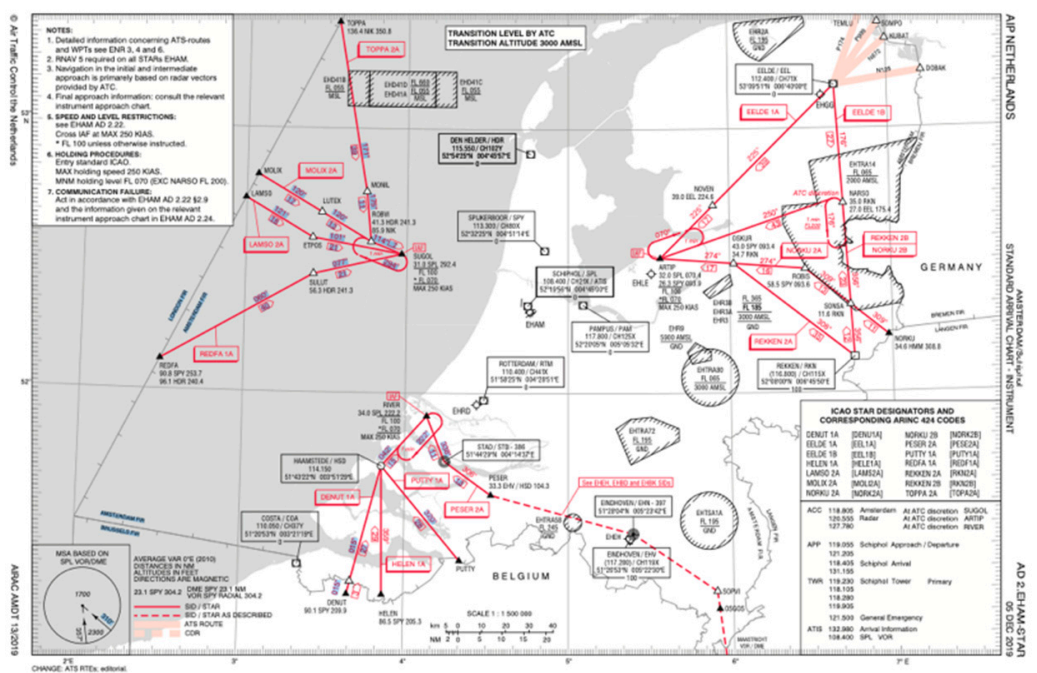

Figure 6. Standard arrival chart at Schiphol Airport. Source [24].

Once approaching and inside Dutch airspace (Milestones 4 and 5), an intense transition of information takes place to update different inbound time references, this data is transmitted mainly in forms of dome messages such as FUM, OLDI-messages, radar and, ACARS data. This information is constantly received and used to trigger a diverse time reference estimation as described in the previous section. In addition to the ELDT, the so-called Estimated In-Block time (EIBT), equivalent to the 
airline/handler Estimated Time of Arrival (ETA) is also updated. These estimations are performed by different IT systems and decision support tools of controllers such as the arrival manager at Schiphol called ASAP and the Amsterdam Advanced Air traffic control system (AAA) is the LVNL radar data and flight data processing system; its primary purpose is to support the air-traffic controllers (ATC) to efficiently and safely manage air traffic. The ELDT at Schiphol is mainly triggered by AAA and ASAP and share through CIISS following selection rules based on the accuracy of the ELDT produced.

In this regard, the aim of a research work found in [25] is to improve both the turn round and outbound planning process at Schiphol mainly based on the fact that the stability of those planning processes depends heavily on the accuracy of the inbound flight information. It has been found that the ELDTs depend on the following main factors: origin of the flight (i.e., initial approach segment used to land), business rules applied to prioritize inbound source of information, and availability of inbound data, the logic implemented on AAA and ASAP to calculate it, ATC manual intervention and procedures used, pop-up flights, and other adverse un-predicted factors such as adverse weather conditions or overloaded surrounding airspace.

Other research regarding the inbound phase can be found in [26] where the new arrival management system at Schiphol called the Advanced Schiphol Arrival Planner (ASAP) was evaluates after being implemented in 2018. To validate the implementation of ASAP diverse analysis have been run. This work describes some recent insights and findings regarding the performance and interaction between users (ATCs) and ASAP. Particular attention is given to establishing the moment and type of interaction between the different users. The performance of the tool was evaluated using four main performance indicators; Expected Approach Time (EAT) adherence, slot adherence, holding ratio, influence of APLN.

\section{Conclusions and Further Work}

This work centers its attention on describing the main elements of A-CDM as a baseline for understanding how common agreed benefits can be monitor. Special attention is placed on the Milestone approach to show the benefits of a standardizing process and vocabulary. This standardization allows us to harmonize operations and vocabulary across Europe and even worldwide and, in addition, to leverage experience gained and lessons learned from airports with similar capacity constraints or infrastructure working under the CDM principles. Moreover, enhancing the use of this terminology will help to develop a common protocol of communication which can be easily extended internationally, allowing benchmark studies among the airports systems as a network of the European network to be performed.

To properly select the main indicators to monitor capacity, a literature review was conducted on two main topics, performance-based approaches and capacity indicators, this is the baseline for the proposed KPIs and performance approach.

It has been emphasised that the milestone approach and information-sharing elements on the CDM principles, set proper a mechanism to evaluate strategic objective agreed by partners in a straightforward and transparent manner. Hence a detail description of the main time reference used on the landing and take-off cycle are presented as part of the background required to monitor operations. In addition, a framework was presented on how to monitor the accuracy of expected capacity on six main moments on time: at entering Terminal Maneuvering Area (TMA) and En-route, at landing and take-off and at in-block and off-block. The expected benefits of the monitoring framework are gaining understanding of the performance of operations on key moments represented by the milestones and time references.

Analyzing the Accuracy of operations will allow mangers and decision makers to take efficient decisions in order to improve overall system capacity by addressing key moments on the LTOC, as in the Milestone Approach. Using the same vocabulary, parameters, principles, and system's conditions, it can be generated a standardized framework to monitor strategic objectives on different time horizons. Schiphol was used as a case study to show how a full A-CDM airport can monitor capacity in the 
different phases of the flight and at different planning horizons (strategic and pre-tactical). It was also selected due to the different efforts which have been performed by its partners. Hence, the proposed framework is intended to be generic, and appropriate for any airport which had implemented CDM principles.

As future work, a more detailed literature review together with interviewing the partners involved in order to properly select the most suitable indicators for their purposes is proposed when selecting the KPIs. Furthermore, a set of actions has to be proposed to control the course of actions during the monitoring process, hence, further analysis has to be performed to propose and select the most appropriate control actions which will be used by decisions makers. Other indicators can also be developed following the same premises presented on this work related to the other strategic objectives.

Author Contributions: C.Z. and G.B. devised the project, the main conceptual ideas and proof outline. Both worked out almost all of the technical details, and verified the ideas presented together with the discussions of the manuscript. All authors have read and agreed to the published version of the manuscript.

Funding: This research received no external funding.

Acknowledgments: The research leading to the results has received funding and information from the Knowledge and Development Centre Mainport Schiphol and Amsterdam University of Applied Sciences.

Conflicts of Interest: The authors declare no conflict of interest.

\begin{tabular}{|c|c|}
\hline \multicolumn{2}{|c|}{ Abbreviations } \\
\hline A-CDM & Airport Collaborative Decision Making \\
\hline AIBT & Actual In-Block Time \\
\hline AOBT & Actual Off-block time \\
\hline ARDT & Actual Ready Time \\
\hline ASAT & Actual Start-Up Approval Time \\
\hline ATOT & Actual Take Off Time \\
\hline ATA & Actual Time of Arrival \\
\hline ATD & Actual Time of Departure \\
\hline ATC & Air Traffic Control \\
\hline ATFCM & Air Traffic Flow and Capacity Management \\
\hline ATFM & Air Traffic Flow Management \\
\hline ATM & Air Traffic Management \\
\hline A-CDM & Airport Collaborative Decision Making \\
\hline $\mathrm{AO}$ & Airport Operators \\
\hline CANSO & Civil Air Navigation Services Organisation \\
\hline СTOT & Calculated Take-Off Time \\
\hline LVNL & Dutch Air Navigation Service Provider \\
\hline ELDT & Estimated Landing Time \\
\hline EXIT & Estimated taxi-in time \\
\hline EXOT & Estimated taxi-out time \\
\hline FIR & Flight Information Region \\
\hline FUM & Flight Update Message \\
\hline $\mathrm{GH}$ & Ground Handlers \\
\hline IAF & Initial Approach Fix \\
\hline IFR & Instrumental Flight Rules \\
\hline ICAO & International Civil Aviation Organization \\
\hline KPIs & Key Performance Indicators \\
\hline LTOC & landing and take-off cycle \\
\hline NMOC & Network Manager Operations left \\
\hline OTP & On-time performance \\
\hline PBAM & Performance Based Airport Management \\
\hline PBM & Performance Based Management \\
\hline PIs & Performance Indicators \\
\hline
\end{tabular}




$\begin{array}{ll}\text { SESAR } & \text { Single European Sky ATM Research } \\ \text { TOBT } & \text { Target Off-Block Time } \\ \text { TSAT } & \text { Target Start-up Approval Time } \\ \text { TTOT } & \text { Target Take Off Time } \\ \text { TMA } & \text { Terminal Maneuvering Area } \\ \text { TAM } & \text { Total Airport Management } \\ \text { VTT } & \text { Variable Taxi Time }\end{array}$

\section{References}

1. Eurocontrol. Airport Collaborative Decision-Making (A-CDM) Implementation Manual, v5.0; Eurontrol: Brussels, Belgium, 2017.

2. Eurocontrol. Airport CDM-Steps to Boost Efficiency; Eurontrol: Brussels, Belgium, 2009.

3. Schipol. Schiphol Webpage. 2020. Available online: https://www.schiphol.nl/en/ (accessed on 31 January 2020).

4. Artley, W.; Ellison, D.; Kennedy, B. The Performance-Based Management Handbook; Oak Ridge Associated Universities: Oak Ridge, TN, USA, 2001.

5. Franceschini, F.; Galetto, M.; Maisano, D. Management by Measurement: Designing Key Indicators and Performance Measurement Systems; Springer Science \& Business Media: Berlin, Germany, 2007.

6. Milanovic Glavan, L.J. Understanding Process Performance Measurement Systems; Technical Report No.2; Business Systems Research: Zagreb, Croatia, 2011; Volume 2.

7. ICAO. DOC 9854-Global Air Traffic Management Operational Concept; ICAO: Montreal, QC, Canada, 2005.

8. ICAO. DOC 9883-Manual on Global Performance of the Air Navigation System; ICAO: Montreal, QC, Canada.

9. Eurocontrol. Eurocontrol Webpage. 2020. Available online: https://www.eurocontrol.int/project/total-airportmanagement (accessed on 31 January 2020).

10. ICAO. International civil aviation organization, working paper. In Proceedings of the 13th Air Navigation Conference, Montréal, QC, Canada, 9-19 October 2018.

11. Helm, S.; Loth, S.; Schultz, M. Advancing total airport management-An introduction of performance based management in the airport context. In Proceedings of the 19th ATRS World Conference, Singapore, 2-5 July 2015.

12. Helm, S.; Urban, B.; Werner, C.; Grimme, W. Key performance indicators for landside processes at airports-Which to choose and what to gain? In Proceedings of the World Conference of Transport Research, Rio de Janeiro, Brazil, 15-18 July 2013.

13. ICAO. DOC 9882-Manual on Air Traffic Management Systems requirements; ICAO: Montreal, QC, Canada, 2008.

14. Doc D3.1/2017: APACHE Project Deliverable D3.1-Review of Current KPIs and Proposal for New Ones; SESAR Joint Undertaking: Brussels, Belgium.

15. APACHE. Review of Current KPIs and Proposal for New Ones; APACHE Consortium H2020-SESAR-2015-1; APACHE: Houston, TX, USA, 2017.

16. CANSO. Airport Collaborative Decision-Making: Optimisation through Collaboration. In An Introductory Guide for Air Navigation Service Providers; CANSO: Amsterdam, The Netherlands, 2016.

17. Eurocontrol. Airport Capacity Assessment Methodology ACAM Manual v1.1; Eurontrol: Brussels, Belgium, 2016.

18. Schiphol. Schiphol Airport CDM Operations Manual, v1.3; Schiphol: Amsterdam, The Netherlands, 2019.

19. Airport Coordination Netherlands. Capacity Declaration for Amsterdam Airport Schiphol (AMS) for IATA Season Summer 2020 (S20); Airport Coordination Netherlands Schiphol: Amsterdam, The Netherlands, 2019.

20. Voog, M. Operational Benefits Since the Connection of Amsterdam Airport Schiphol with the Network Manager. Knowledge Development Center (KDC) Thesis Report. 2019. Available online: https://kdcmainport.nl/centre-of-excellence/ (accessed on 31 January 2020).

21. Wouters, R. Impact of Local A-CDM on the Operational Efficiency at Mainport Schiphol. Knowledge Development Center (KDC) Thesis Report. 2018. Available online: https://kdc-mainport.nl/centre-ofexcellence/ (accessed on 31 January 2020).

22. Mollema, F. Planning Inefficiencies within the Overall Planning System of Mainport Schiphol. An Analysis of ALIGNMENT issues Between the Major Stakeholders in the Dutch Aviation Sector, Causing Planning Inefficiencies in the Inbound and Turnaround Phase. Knowledge Development Center (KDC) Thesis Report. 2019. Available online: https://kdc-mainport.nl/centre-of-excellence/ (accessed on 31 January 2020). 
23. Wassenberg, F.F. D-1 Prediction. Validating and Improving the Prediction of the Demand at the day Before Operation. Knowledge Development Center (KDC) Thesis Report. 2019. Available online: https://kdc-mainport.nl/centre-of-excellence/ (accessed on 31 January 2020).

24. IVAO. International Virtual Aviation Organisation Webpage. 2020. Available online: https://nl.ivao.aero (accessed on 31 January 2020).

25. Scheffers, T. Validating air Traffic Systems of the LVNL in the Arrival Process of Schiphol. An Analysis of the ELDT. Knowledge Development Center (KDC) Thesis Report. 2019. Available online: https://kdc-mainport.nl/centre-of-excellence/ (accessed on 31 January 2020).

26. Hollebeek, A. Evaluation of AMAN Implementation to Establish Improvements in the Arrival Efficiency. Knowledge Development Center (KDC) Thesis Report. 2019. Available online: https://kdc-mainport.nl/ centre-of-excellence/ (accessed on 31 January 2020).

C 2020 by the authors. Licensee MDPI, Basel, Switzerland. This article is an open access article distributed under the terms and conditions of the Creative Commons Attribution (CC BY) license (http://creativecommons.org/licenses/by/4.0/). 